\title{
APPLICATION OF MOTION CAPTURE SYSTEMS IN ERGONOMIC ANALYSIS
}

\author{
AUTHORS' DATA: \\ Tigran Petrosyan, MD, $\mathrm{PhD}$, Associated professor \\ Department of Kinesiology, Armenian State Institute of Physical Culture and Sports \\ Lecturer \\ Contacts: tigpetrosyan@mail.ru
}

Arayik Dunoyan, MSc, Assistant professor

Department of Kinesiology, Armenian State Institute of Physical Culture and Sports

Lecturer

Contacts: adunoyan@mail.ru

Hasmik Mkrtchyan, MSc, Assistant professor

Department of Kinesiology, Armenian State Institute of Physical Culture and Sports

Lecturer

Contacts: $\underline{\text { hasmikmh@mail.ru }}$

\begin{abstract}
Currently different methods are used for ergonomic assessment and analysis. This review tries to show how motion capture technology is applied in the process of ergonomic assessment. The goals of the analysis were to identify the most adequate method for objective assessment of ergonomics. The results show that the optical motion tracking systems with special software can be used to perform digital analysis of body motion. These systems do not require long set up time, majority of them are portable and the sensors are available in the market for a low cost. Movements of the working person are captured without special clothes equipped with markers. Though the optical systems could be acceptable in a wide range of tasks, they have certain limitations in ergonomic analysis. The performance of optical systems depends on a number of variables such as lighting, type of movements, distance from the object and environmental artefacts. The performance of existing systems is not yet completely reliable, but the technology is on the path of improving its accuracy. There are also other mechanical and magnetic technologies used for
\end{abstract}


ergonomic analysis. This review shows that ergonomic simulations using the motion capture technology significantly improves the quality of ergonomic analysis.

Keywords: Ergonomics, Ergonomic analysis, Motion Capture Technology, Product Development, Work related injuries.

\section{INTRODUCTION}

Ergonomic analysis is widely used in industry and product development. The word has a Greek origin - "ergon" meaning work, and "nomons" - laws. Ergonomists together with engineers evaluate ergonomics to ensure the job safety, develop products, ensure the safety of working environment, abilities and limitations of the employed professionals (IEA, 2006). Though the term ergonomics is perceived in different ways around the world, the International Ergonomic Association (IEA) has introduced a full definition of this term: "Ergonomics is the scientific discipline concerned with the understanding of interactions among humans and elements of a system, and the profession that applies theory, principles, data and methods to design in order to optimize human well-being and overall system performance" (IEA, 2006). The sphere of ergonomics encompasses three different areas: organizational ergonomics, cognitive ergonomics and physical ergonomics. Physical ergonomics studies human anatomical, physiological, anthropometric and biomechanical characteristics. Aspects that the physical ergonomics is concentrated on are the working postures, workplace layout, repetitive movements, work related musculoskeletal disorders, safety and health. The importance of the ergonomic analysis can be shown on the example of vehicle production.

The production of a vehicle starts from its design and the process of design is a complex task with different issues to consider (Högberg, Bäckstrand, Lämkull, Hanson et al., 2008). First of all, the design activities have to ensure that the buyer can use all the necessary functions in a comfortable and safe manner. Designing a vehicle for production requires conditions and capabilities to ensure the large volume of production, recruiting the required number of employees (Faraway \& Reed, 2007). Though the automotive industry is rather advanced, variety of parts and details used for the vehicle production are made and produced manually, which significantly affect the ergonomics (Klippert, Gudehus, Zick, 2012). A large number of studies have shown that proper ergonomics can not only improve the workers' health but also have a positive economic impact on the company (Fritzsche, 2010). A study conducted in 1995 revealed that the rate of quality 
deficiencies identified in the car assembly process was three times higher in work tasks and procedures were ergonomic problems were obvious (Eklund, 1995). It is therefore essential to implement the ergonomic workplace design when starting the layout and facility planning for the designed production, in order to reduce the extra costs in the future production process (Klippert, Gudehus, Zick, 2012). It is highly suggestive to consider the ergonomics in early stages of the product development process, analyzing the interaction of a person with the product. Reduction of the negative impact on human health and consequently on economics can lead to the manufacturing of more sustainable products and sustainable cohort of users in the society.

The process of the vehicle design that fits both the needs of customers and requirements of the workers, considering the possible limitations, is based on the ergonomic assessments performed during the product development process. In the past, this assessment was performed by building a sequence of physical prototypes, that allowed to evaluate the important or necessary parameters (Faraway \& Reed, 2007). Nowadays, the process of product realization is mainly constrained by high cost and time restrictions where the major costs are based on an inefficient physical work (Högberg, Bäckstrand, Lämkull, Hanson et al., 2008). For the recent decades the computer based visualization or simulation methods are widely used for early evaluation, and verification of critical design parameters specific for the ergonomics (Chaffin, 2005; Klippert, Gudehus, Zick, 2012). For this purpose, digital human models (DHM) are used by design engineers in early stages of product development processes. These models allow engineers to simulate the user performing a specific task or movement. DHMs are implemented in the design of aircraft, cars and trucks (Faraway \& Reed, 2007). These so called manikins are widely used in the vehicle industry to improve the interior design and manufacturing workplaces (Chaffin, 2005). This touches also the question of physical ergonomics for assembly workers. Research evidence indicates that DHMs can reduce the engineering costs and have to be implemented in the early development process (Chaffin, 2005; Klippert, Gudehus, Zick, 2012). By reducing the production time and costs, these technologies increase the profitability, as well as the product quality (Högberg, Bäckstrand, Lämkull, Hanson et al., 2008). DHM are used not only in the industry or the engineering field. They are applied as research tools in neuroscience, biomechanical analyses (e.g. gait studies), sports performance analysis, in the movie and videogame production, and other relevant fields (Faraway \& Reed, 2007; Jung, Zhou, Ramsey \& Krovi, 2013). Depending on the purpose of use, the type and functions of these systems differ (Jung, Zhou, Ramsey \& Krovi, 2013). 
Before setting up a DHM analysis, the specialist has to specify the population and other anthropometric data that are peculiar for the user, position and posture relevant for the simulation [1]. The way people move and interact in a vehicle or workplace environment is extremely versatile, depending on their actual physical size, age, gender, and other parameters. Naturally the human behavior is different in the same setting and difficult to predict (Faraway \& Reed, 2007).

\section{THE BODY}

The review tries to show possible application of motion capture systems in ergonomic analysis and injury prevention. For this reason, separate subunits of the article present the functional capabilities of motion capture systems and some aspects and specifics of work related injuries.

\section{MOTION CAPTURE TECHNOLOGY}

Motion capture technology is used to register motions of objects and people in variety of settings and for different purposes. The captured and registered data can be used digitally in many different ways (Field, Pan, Stirling \& Naghday, 2011). For the last few decades' motion capture has been widely used in the film making industry to create realistic 3D movements of animated characters (Faraway \& Reed, 2007). In research the motion capture has also been widely used for biomechanical analysis in sports medicine, rehabilitation medicine and athletic training (Pfister, West, Bronner \& Noah, 2014). As mentioned above for over a quarter century the motion capture is used as an analysis tool for the design of different products and ergonomics analysis. In ergonomic analysis the motion capture tools have the capability to perform numerous simulations of the production process and to virtually analyze scenarios where manufacturing operations are performed (Jung, Zhou, Ramsey \& Krovi, 2013). Advanced tracking algorithms used in motion capture and data processing have led to maximum decrease in measurement errors (Field, Pan, Stirling \& Maghday, 2011). Software used with the motion capture systems usually provides accurate analysis. But the technology itself and the software are very costly and not always available in industrial settings where they are of great value (Pfister, West, Bronner \& Noah, 2014). In general, the ergonomic evaluations are based on performance analysis of the working person accomplishing a specific task. The analysis has to be conducted by an ergonomically trained specialist to obtain a full overview of the task. After the registration, the gathered data has to be 
analyzed scrupulously. Motion capture technology provides a more detailed analysis than any other human observation can produce, with the possibility to register 30 to 120 frames per second. Usually, the motion capture setup time is about $20 \mathrm{~min}$, but still this method is considered more efficient than any other tool or technology (Klippert, Gudehus, Zick, 2012). However, there is no a unique opinion about the motion capture systems and digital human models. According to Chaffin (2005) the posture analysis of the studied subject should not be used together with captured motion analysis; the software should do that for you in different modes and. Simulating a very simple task, such as walking to a table and picking up a tool, can require time consuming manual posturing at each critical transition point of the performed motion. This kind of animation process can require from the analyst at least several hours to simulate a task that would only take a few seconds for the individual to perform.

Three main types of tracking methods have been developed during the decades of motion capture development; mechanical, optical and magnetic systems (Faraway \& Reed, 2007). Each method has its strengths and weaknesses, based on the purpose of the use. The optical system requires application of reflective markers, that are placed on specific hallmarks of the person performing the specific task or the studied motion. These target markers are then captured and triangulated by several registering cameras using infrared (IR) light. Before motion can be tracked, the fixed markers have to be identified by the computer software, named by the investigator (such as left shoulder, right shoulder and so on). However, some active optical systems offer special markers that do not require pre-identification (Faraway \& Reed, 2007). The accuracy of these type of optical systems is often very good, with a possible error of one millimeter or less. This kind of tracking process is rather time consuming and the precise positioning of the markers are essential. If a marker is misplaced, slips or becomes blocked, the tracking will be inaccurate. Motion capture systems like this are mostly limited to a lab setting and are used for the research purposes (Faraway \& Reed, 2007). Qualisys is a Swedish company that specializes in motion capture, providing highprecision optical motion capture systems to clients over the world. Qualisys systems are applied in different fields, for example in medical research, where doctors and physiotherapists can improve treatment by obtaining a better understanding of the biomechanics captured by the motion capture system. The same systems are also being used in industrial settings, sports science, media and entertainment. 


\section{Picture 1. Motion capture system for the ergonomic analysis}

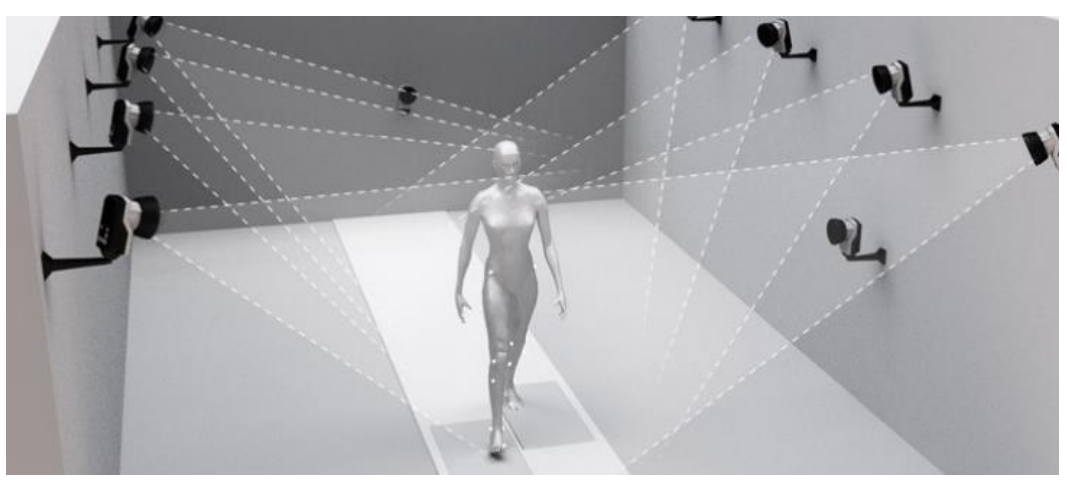

The other type of motion capture system - the magnetic system consists of three orthogonal coils installed in a transmitter. The relative magnetic flux between the transmitter and receiver allows to determine the location and orientation of the receiver. The markers are placed on the exterior of the body, whereas in other motion capture systems the markers are placed on central points of joints (Faraway \& Reed, 2007). Compared to optical motion capture systems, magnetic systems have certain advantages when the motion capture is performed in narrow spaces. Although almost all magnetic systems require markers to be wired, restricting the motion and interfering with the movements of the subject. Another disadvantage of the magnetic systems is their sensitivity to metallic objects in the environment (this is more critical in industrial settings), as well as to electrical fields produced by other experimental equipment (Faraway \& Reed, 2007; Haggag, Hossny, Haggag, Nahavandi, et al., 2014). Polhemus is a well-known prototype of the magnetic tracking system that has been used within the military since 1969.

Mechanical systems can capture motion through a set of goniometers or accelerators that are fixed on the subject, either using separate goniometers or a full suit with variety of registering tools. When the subject is moving, these goniometers are registering the motion, and the data is sampled and stored in a device. Mechanical systems offer an advantage that the other systems lack: they do not interfere with light or magnetic fields, and they don't need to be calibrated before each registration, which is very time consuming. This type of mechanical system was used to produce the animated movies.

\section{WORK RELATED INJURIES}

Work injuries and ergonomic analysis individuals involved in professional activities aims to reduce the rate of injuries. The best example can be the trucking Industry. Truck drivers are 
frequently injured, and other than the accidents slip and falls in their work are the most common reasons for work related injuries (TSWEA, 2014). In 2003, the number of work injuries that lead to lost workdays was twice higher in truck drivers compared to the average of all professions (not only the drivers) in Sweden, and about half of these work related injuries resulted in the sick leave for longer than two weeks (TSWEA, 2014). The most common locations of fall accidents have been reported to be at the back of the truck (49.1\%), the steps (18.5\%) and the cargo (16.5\%) [9] and six out of ten falls were from a higher distance than one meter above the ground (Jones \& Switzer - McIntyre, 2003; TSWEA, 2014). The most common types of injuries due to slips and falls were strains/sprains, contusions, abrasions and fractures (Jones \& Switzer - McIntyre, 2003). In the study conducted by Jones and Switzer-McIntyre the most common areas of body injured during work were lumbar (19.7\%), shoulder (9.7\%), wrist $(9.3 \%)$ and knee $(8.2 \%)$ (Jones \& Switzer - McIntyre, 2003). In a study, conducted in the US, 3053 cases of work related injuries were analyzed, from which 277 cases (9\% of the total cases) were accidents caused by the ingress/egress. The primary causes of these injuries were wet steps because of rain, truck washing, snow or ice built up on or at the steps. On the other hand, the results presented by Jones and Switzer-McIntyre showed that $79 \%$ out of the all reported fall cases were not caused by environmental factors (Jones \& Switzer - McIntyre, 2003). It is noteworthy that from all cases of fall injuries there were three times more egress than ingress accidents. Injuries caused by work environment are a substantial cost to companies and employers, as well as to employees (Jones \& Switzer - McIntyre, 2003). The high risk of injuries among the track drivers forced to implement ergonomic analysis in the truck development process. Ingress/egress has been a central issue in ergonomic analysis and development of the cabs. For example, this aspect is extensively developed by the Scania's department of ergonomics to reduce the risks of injuries.

By capturing motion of employees, a large volume of information could be obtained that cannot be gained by simple visual observation. The motion capture records joint angles and exact positioning of body parts transforming them into a digital format. The digital data is saved, has an easy access for further analysis. The data output is used in different ways: identification of critical joint angles that may lead to an increased risk of injury, verification of fulfilled ergonomic recommendations. However, the digital human models with simulating movements have been shown to be extremely time consuming. One of the mechanisms to reduce the preparation and simulation time is the registration of actual movements or procedure by the motion capture system, 
then step by step designing a simulated movement model. In case of the ergonomic analysis performed for the truck drivers, movements are recorded in relation to a simplified model of the cab. The recorded chain of movements is later transformed to a digital human model and compared to the actual model of the newly implemented design concept. This approach is applied to test the new design concepts when there is a need to evaluate the possibility in which manikin would collide with objects, or this method might be used to evaluate if different objects inside the cabin or out of it are within a driver's reach.

\section{Picture 2. laboratory setting for the ergonomic assessment}

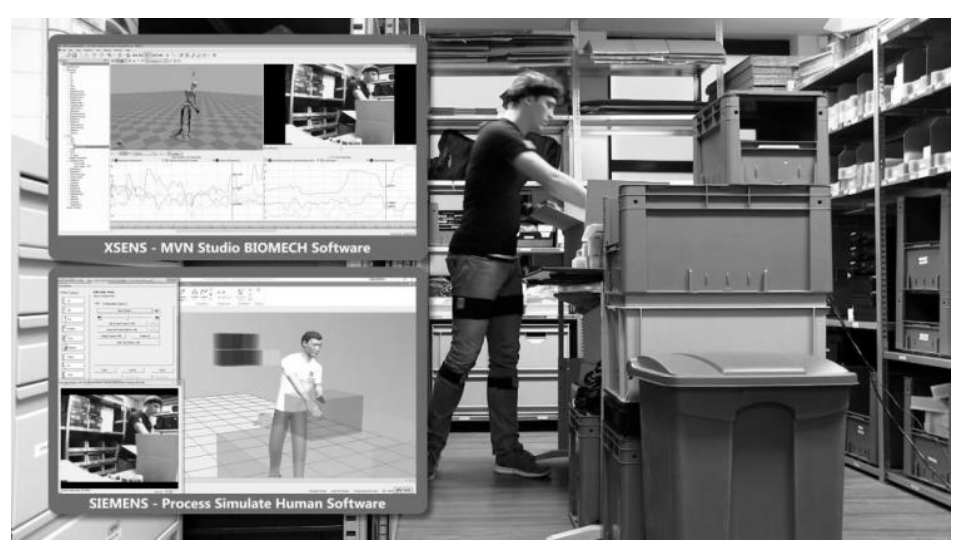

This approach can lead to much earlier verifications of ergonomics, which is able to save time and resources, compared to the other classic DHM simulations used nowadays. Search of the research databases has revealed overwhelming number of research studying the truck driver ergonomics and the incidence of work related injuries (TSWEA, 2014). Implementing the cab that has different structure and design may decrease the incidence of injuries, and motion captured could be the best method for identifying the issues related to the ingress. The goal of visual analysis could be the different positions of cab steps that affect the way drivers place their foot on steps. Egress accidents and rate of egress injuries are three times higher compared to ingress injuries. Lack of visibility in egress process might be the primary cause of these injuries. It is possible to conduct a visibility test using the virtual reality (VR) glasses. When wearing a pair of VR-glasses, the individual experiences the computer aided design environment in 3D. Using together the motion capture and virtual reality, researchers are able to get a better idea of how a user would react or move when interacting with the new environment. Systems like Kinect and IMMA can provide a quick method for motion capture and analysis. They have been extensively used in facilities of Scania, without any other expensive equipment or technology. On the other hand, more 
detailed analysis could be performed with a more sensitive and accurate system if required, only in a well-equipped research lab.

Based on the research evidence a question arises: which motion capture systems to use in the industry and ergonomic analysis. When looking for motion capture systems that are adequate for the truck cab development, there are different options to choose between. Other than the Kinect sensor and IMMA software there are many other systems. The type of the system applied depends on the purpose of the study or the goal of applied evaluation. In trials where the goal is to capture the exact positioning of the subject with accuracy of $1 \mathrm{~mm}$ or less, the motion capture should be performed by advanced optical systems. The only disadvantage of these systems is the cost. These systems are very expensive, require expert knowledge of the human body and placement of markers in the right location. The industries and manufacturers that don't have their own research units are trying to collaborate with labs that have the technology and experience in using motion capture systems. Though the optical systems are more advanced than the other technology they not always fit the goals or interests of the industrial company. There are certain limitations for optical systems when registering a task inside the cab. The limitations are due to the restrictions regarding a free line of sight. In such cases, mechanical systems are a better option as they do not depend on the free line of sight. The mechanical systems have advantages when capturing movements while driving. The applied mechanical sensors and goniometers are placed on the parts of the body that are involvement in the specific task performance.

\section{CONCLUSION}

Motion capture technology has potential to serve as a comprehensive tool for ergonomic assessments used for the product development process in vehicle industry, or other fields. Motion capture technology enables registration of movements in 3D mode, which is not possible to gain by only observing a motion, or registering it with other methods and simple cameras. Recording the joint angles and precise positioning of body parts is easily transformed into a digital format and saved for further assessment and analysis. The data processing can provide the critical joint angles that possess an increased risk for injury, or to verify that all ergonomic recommendations are properly followed. Motion capture recording is based on data collected from the real human movements and behavior, providing an objective background for comparison and analysis. Simulation models require a long set up time, whereas motion capture enables to track the motions 
rapidly and analyze them in a digital format. Different design concepts can be tested using this technology, providing an early verification of ergonomic requirements, and decreasing the production and health related costs.

\section{REFERENCE LIST}

1. Chaffin, D. B. (2005). Improving digital human modeling for proactive ergonomics in design. Ergonomics 48(5) 478 - 491

2. Eklund, J. (1995). Relationships between ergonomics and quality in assembly work. Applied Ergonomics, 26(1), 15-20. doi:10.1016/0003-6870(95)95747-N

3. Faraway, J. \& Reed, M. P. (2007). Statistics for Digital Human Motion Modeling in Ergonomics. Technometrics, 49(2), 277-290. doi:10.1198/004017007000000281

4. Field, M., Pan, Z., Stirling, D. \& Naghday, F. (2011). Human motion capture sensors and analysis in robotics. Industrial Robot: An International Journal 38(2) 163-171

5. Fritzsche, L. (2010). Ergonomics Risk Assessment with Digital human Models in Car Assembly: Simulations versus Real Life. Human Factors and Ergonomics in Manufacturing \& Service Industries, 20(4), 287-299. doi:10.1002/hfm.20221

6. Haggag, H., Hossny, M., Haggag, S., Nahavandi, S. \& Creighton, D. (2014). Safety applications using Kinect technology. In IEEE International Conference on Systems, Man, and Cybernetics 2014. San Diego, CA. October 5-8, 2164-2169

7. Högberg, D., Bäckstrand, G., Lämkull, D., Hanson, L. \& Örtengren, R. (2008). Industrial customisation of digital human modeling tools. Int. J. Services Operations and Informatics 3(1),53-70

8. International Ergonomic Association (2006). What is ergonomics? Retrieved on 2014-1212 from: http://www.iea.cc/

9. Jones, D. \& Switzer-McIntyre, S. (2003). Falls from trucks: A descriptive study based on a workers compensation database. IOS Press, 20 (2003), 179-185

10. Jung, S-K., Zhou, X., Ramsey, D.K. \& Krovi, V.N. (2013). A Comparison Study of Human Motion Capture and Computational Analysis Tools. Buffalo, New York.

11. Klippert, J., Gudehus, T. \& Zick, J. (2012). A Software-Based Method for Ergonomic Posture Assessment in Automotive Preproduction Planning: Concordance and Difference 
in Using Software and Personal Observation for Assessments. Human Factors and Ergonomics in Manufacturing \& Service Industries 22(2) 156-175

12. Pfister A., West, A.W., Bronner, S. \& Noah J. A., (2014). Comparative abilities of Microsoft Kinect and Vicon 3D motion capture for gait analysis. Journal of Mecanical Engineering \& Technology 38(5), 274-280.

13. The Swedish Work Environment Authority (2014). Transportbranschen. Korta arbetsfakta, Nr 2. The Swedish Work Environment Authority (2004). Last- och långtradarförare. Korta sifferfakta, Nr 3 